\title{
Some families of 0-rotatable graceful caterpillars
}

\author{
Atílio G. Luiz ${ }^{1}$, C. N. Campos ${ }^{1}$, R. Bruce Richter ${ }^{2}$ \\ ${ }^{1}$ Instituto de Computação - Universidade Estadual de Campinas (UNICAMP) \\ Campinas - São Paulo - Brasil \\ ${ }^{2}$ Department of Combinatorics and Optimization - University of Waterloo \\ Waterloo - Ontario - Canada \\ gomes.atilio@gmail.com, campos@ic.unicamp.br, brichter@uwaterloo.ca
}

\begin{abstract}
A graceful labelling of a tree $T$ is an injective function $f: V(T) \rightarrow$ $\{0,1, \ldots,|E(T)|\}$ such that $\{|f(u)-f(v)|: u v \in E(T)\}=\{1,2, \ldots,|E(T)|\}$. A tree $T$ is said to be 0-rotatable if, for any $v \in V(T)$, there exists a graceful labelling $f$ of $T$ such that $f(v)=0$. In this work, it is proved that the following families of caterpillars are 0-rotatable: caterpillars with perfect matching; caterpillars obtained by identifying a central vertex of a path $P_{n}$ with a vertex of $K_{2}$; caterpillars obtained by identifying one leaf of the star $K_{1, s-1}$ to a leaf of $P_{n}$, with $n \geq 4$ and $s \geq\left\lceil\frac{n-1}{2}\right\rceil$; caterpillars with diameter five or six; and some families of caterpillars with diameter at least seven. This result reinforces the conjecture that all caterpillars with diameter at least five are 0-rotatable.
\end{abstract}

\section{Introduction}

Let $G=(V(G), E(G))$ be a graph with vertex set $V(G)$ and edge set $E(G)$. A graceful labelling of $G$ is an injection $f: V(G) \rightarrow\{0,1, \ldots,|E(G)|\}$ such that $\{\mid f(u)-$ $f(v) \mid: u v \in E(G)\}=\{1,2, \ldots,|E(G)|\}$. We say that $G$ is graceful if it has a graceful labelling.

In 1967, Rosa introduced four types of labellings of graphs, including graceful labellings, and posed the Graceful Tree Conjecture which states that all trees are graceful [Rosa 1967]. Rosa proved that the Graceful Tree Conjecture is a strenghtened version of the well-known Ringel-Kotzig Conjecture which states that the complete graph $K_{2 m+1}$ has a cyclic decomposition into subgraphs isomorphic to a given tree $T$ with $m$ edges. The Graceful Tree Conjecture is a very important open problem in Graph Theory, with more than a thousand papers about it [Gallian 2015].

As soon as one starts investigating graceful labellings of trees, it becomes clear the importance of knowing how to construct graceful labellings with the label 0 appearing in a given vertex. The importance of label 0 in a graceful labelling of a tree $T$ is due to the fact that it is easy to grow $T$ by adding $k$ new leaves to the 0-labelled vertex and expand the graceful labelling by assigning labels $|E(T)|+1, \ldots,|E(T)|+k$ to these new leaves. A tree $T$ is 0 -rotatable if, for any $v \in V(T)$, there exists a graceful labelling $f$ of $T$ such that $f(v)=0$.

The importance of 0-rotatability of trees was first noted by Rosa in his seminal paper [Rosa 1967], in which the author stated, without proof, that all paths are 0-rotatable. Ten years later, the author published a proof of this result [Rosa 1977]. Meanwhile, in 1969, some examples of non 0-rotatable trees were discovered [Gallian 2015]. As an 
example, the smallest non 0-rotatable tree is the tree obtained by identifying one leaf of the star $K_{1,3}$ to a leaf of $P_{3}$. Posteriorly, Chung and Hwang investigated the 0-rotatability of a product of trees called $\Delta$-construction and proved that if two trees $T_{1}$ and $T_{2}$ are 0 rotatable, then their product $T_{1} \Delta T_{2}$ is also 0-rotatable [Chung and Hwang 1981]. In 2004, Bussel [Bussel 2004] showed that all trees with diameter at most three are 0-rotatable. The author also showed that there exist non 0-rotatable trees with diameter four. In fact, he completely characterized the diamater-four non 0-rotatable trees using the following result.

Theorem 1 ([Bussel 2004]). Let $T$ be a tree of diameter four such that its center $v$ has degree two. Let $v_{1}, v_{2}$ be the vertices adjacent to $v$ and $m_{1}, m_{2}$ be the number of leaves adjacent to $v_{1}, v_{2}$, respectively. Assume $m_{1} \geq m_{2}$. The tree $T$ has a graceful labelling $f$ with $f(v)=0$ if and only if there exist integers $x$ and $r$ such that $m_{1}=\left(m_{2}+2-\right.$ $x)(r-1)-x$, with: (i) $x, r$ not both odd; (ii) $2 \leq r \leq|E(T)| / 2$; and (iii) $0 \leq x \leq$ $\min \left\{r-1, m_{2}\right\}$.

Let $\mathcal{D}$ denote the class of diameter-four trees whose center has degree two and that do not satisfy the conditions of Theorem 1 . Let $\mathcal{D}^{\prime}$ be the class of trees built by identifying a leaf of an arbitrary path $P_{n}, n \geq 1$, with the center of a tree in $\mathcal{D}$. Bussel proved that, given a tree $T$ with diameter four, $T$ is 0 -rotatable if and only if $T \notin \mathcal{D}^{\prime}$. Additionally, he showed that all trees with at most 14 vertices and that are not 0 -rotatable belong to the class $\mathcal{D}^{\prime}$. Thus, based on these results, the author posed the following conjecture.

Conjecture 2 ([Bussel 2004]). The class $\mathcal{D}^{\prime}$ contains all non 0-rotatable trees.

From the time it was first studied, 50 years ago, 0-rotatability of trees has been considered a possible way to approach the Graceful Tree Conjecture, and also a challenging problem by itself. In particular, a family of trees for which the 0-rotatability property is not known is the family of caterpillars, defined as follows. A tree $T$ is a caterpillar if either $T$ is a path or the subgraph obtained by deleting all its leaves (the base of $T$ ) is a path.

In fact, note that, if Conjecture 2 is true, then it implies that every caterpillar with diamater at least five is 0-rotatable. Considering these observations, in this work, we investigate Conjecture 2 restricted to caterpillars and prove that the following families of caterpillars are 0-rotatable: (i) caterpillars with perfect matching; (ii) caterpillars obtained by identifying a central vertex of $P_{n}$ with a vertex of $K_{2}$; (iii) caterpillars obtained by identifying one leaf of $K_{1, s-1}$ to one leaf of $P_{n}$, with $n \geq 4$ and $s \geq\left\lceil\frac{n-1}{2}\right\rceil$; (iv) caterpillars with diameter five or six; and (v) some families of caterpillars with diameter at least seven. These results reinforce Conjecture 2.

\section{Preliminaries}

A matching of a graph $G$ is a set of pairwise nonadjacent edges of $G$. Let $M$ be a matching of a graph $G$. A vertex $v \in V(G)$ is saturated by $M$ if $v$ is incident with an edge of $M$. A perfect matching of $G$ is a matching that saturates all the vertices of $G$. Let $T$ be a tree with a perfect matching $M$. The contree of $T$ is the tree $T^{\prime}$ obtained from $T$ by contracting all the edges of $M$.

Broersma and Hoede [Broersma and Hoede 1999] introduced the concept of strongly graceful labellings of trees defined as follows. Let $T$ be a tree with a perfect 
matching $M$. A labelling $f$ of $T$ is strongly graceful if $f$ is a graceful labelling and if $f(u)+f(v)=|E(T)|$ for every edge $u v \in M$. The authors proved that the Graceful Tree Conjecture is true if and only if every tree with a perfect matching has a strongly graceful labelling. They also studied the label 0 in strong graceful labellings, as presented in the next lemma. This result is important for the proof of Theorem 5.

Lemma 3 ([Broersma and Hoede 1999]). Let $T$ be a tree with a perfect matching $M$ and $u v \in M, u, v \in V(T)$. Let $T^{\prime}$ be the contree of $T$ and let $x \in V\left(T^{\prime}\right)$ be the vertex corresponding to the edge uv. If $T^{\prime}$ has a graceful labelling $f^{\prime}$, with $f^{\prime}(x)=0$, then $T$ has two strongly graceful labellings $f_{1}$ and $f_{2}$, such that: $(i) f_{1}(u)=0$ and $f_{1}(v)=|E(T)|$; (ii) $f_{2}(u)=|E(T)|$ and $f_{2}(v)=0$.

Given a graceful labelling $f$ of a tree $T$, the complementary labelling of $f$ is the labelling $\bar{f}$ defined by $\bar{f}(v)=|E(T)|-f(v)$ for each $v \in V(T)$. Note that the complementary labelling is also a graceful labelling since: (i) $f(v)$ is an injection from $V(T)$ to $\{0, \ldots,|E(T)|\}$; and (ii) for each $u v \in E(T),|\bar{f}(u)-\bar{f}(v)|=\mid(m-f(u))-$ $(m-f(v))|=| f(v)-f(u) \mid$.

A technique used in our proofs is the method of transfers, defined as follows. Let $u, v, u_{1}$ be distinct vertices of a tree $T$, such that $u_{1}$ is adjacent to $u$. We call transfer, the operation of deleting the edge $u_{1} u$ from $T$ and adding the edge $u_{1} v$. After the transfer operation, we say that $u_{1}$ has been moved from $u$ to $v$. The following lemma determines when a transfer performed over a graceful tree generates another graceful tree.

Lemma 4 ([Hrnčiar and Haviar 2001]). Let $f$ be a graceful labelling of a tree $T$ and let $u, v \in V(T)$ be two distinct vertices. If $u$ is adjacent to leaves $u_{1}, u_{2} \in V(T)$, such that $u_{1} \neq v, u_{2} \neq v$ and $f\left(u_{1}\right)+f\left(u_{2}\right)=f(u)+f(v)$, then the tree $T^{\prime}$ obtained by moving $u_{1}, u_{2}$ from $u$ to $v$ is also graceful.

\section{Results}

In this section, we state our main results. In particular, Theorems 7 and 8 , and the second family stated in Theorem 6 show that, for each integer $d \geq 5$, there exist 0-rotatable caterpillars with diameter $d$ and an arbitrarily large number of vertices. These results reinforce the conjecture that all caterpillars with diameter at least five are 0-rotatable.

Theorem 5. Every caterpillar with a perfect matching is 0-rotatable.

Proof. Let $T$ be a caterpillar with a perfect matching $M$ and let $u v \in M, u, v \in V(T)$. Let $T^{\prime}$ be the contree of $T$ and let $x \in V\left(T^{\prime}\right)$ be the vertex corresponding to the edge $u v$. Since $T$ has a perfect matching, we have that $T^{\prime}$ is a path. Rosa proved that every path is 0-rotatable [Rosa 1977]. Therefore, $T^{\prime}$ is 0-rotatable. Hence, $T^{\prime}$ has a graceful labelling $f^{\prime}$ such that $f^{\prime}(x)=0$. By Lemma $3, T$ has two strongly graceful labellings $f_{1}$ and $f_{2}$ such that: $f_{1}(u)=0$ and $f_{1}(v)=|E(T)| ; f_{2}(u)=|E(T)|$ and $f_{2}(v)=0$. Therefore, there exist strongly graceful labellings of $T$ which assign the label 0 to vertex $u$ or $v$. Since $u v$ is arbitrary, we conclude that $T$ is 0 -rotatable.

Theorem 6. The following families of caterpillars are 0-rotatable: (i) caterpillars obtained by identifying a vertex of $K_{2}$ with a central vertex of $P_{n}$; (ii) caterpillars obtained by identifying one leaf of the star $K_{1, s-1}$ to a leaf of $P_{n}$, with $n \geq 4$ and $s \geq\left\lceil\frac{n-1}{2}\right\rceil$.

Outline of the proof. Let $T$ be a caterpillar as defined in the hypothesis and let $v \in V(T)$ be an arbitrary vertex. First, we specify an edge $w z \in E(T), w, z \in V(T)$, and remove 
$w z$ from $T$, thus obtaining two vertex-disjoint subgraphs $H_{1} \subset T$ and $H_{2} \subset T$ such that $w, v \in V\left(H_{1}\right)$ and $z \in V\left(H_{2}\right)$. Consider a bipartition $\left\{V_{1}, V_{2}\right\}$ of $V\left(H_{1}\right)$ such that $v \in V_{1}$ and define $k=\left|V_{1}\right|$. Thus, we construct injective labellings $f_{1}, f_{2}$ for $H_{1}$, $H_{2}$, respectively, where $f_{1}: V\left(H_{1}\right) \rightarrow\{0, \ldots, k-1\} \cup\left\{k+\left|E\left(H_{2}\right)\right|+1, \ldots,|E(T)|\right\}$, $f_{2}: V\left(H_{2}\right) \rightarrow\left\{k, k+1, \ldots, k+\left|E\left(H_{2}\right)\right|\right\}$, and such that: (i) $f_{1}(v)=0$; (ii) the edge labels induced by $f_{2}$ are $1,2, \ldots,\left|E\left(H_{2}\right)\right|$; (iii) the edge labels induced by $f_{1}$ are $\left|E\left(H_{2}\right)\right|+$ $2, \ldots,|E(T)|$; and (iv) $f_{1}(w)$ and $f_{2}(z)$ are such that $\left|f_{1}(w)-f_{2}(z)\right|=\left|E\left(H_{2}\right)\right|+1$. Finally, we define a labelling $f$ of $T$ as follows: for $u \in V(T), f(u)=f_{1}(u)$ if $u \in H_{1}$; and $f(u)=f_{2}(u)$ if $u \in H_{2}$. Therefore, $f$ is a graceful labelling of $T$ with $f(v)=0$ and, since $v$ is an arbitrary vertex, we obtain that $T$ is 0-rotatable.

Theorem 7. If $T$ is a caterpillar with diameter five or six, then $T$ is 0-rotatable.

Outline of the proof. Let $T$ be a caterpillar with diameter five or six. For each vertex $v \in V(T)$ in the base of $T$, we construct a graceful labelling $f$ of $T$ that assigns label 0 to $v$ and assigns label $|E(T)|$ to any leaf $u \in V(T)$ adjacent to $v$. Consequently, given any of these graceful labellings $f$, one can use its complementary labelling $\bar{f}$ in order to obtain $\bar{f}(u)=0$ and $\bar{f}(v)=|E(T)|$. Since $\bar{f}$ is also a graceful labelling and $f$ was constructed considering an arbitrary vertex $v$ of the base of $T$, we obtain that $T$ is 0 -rotatable.

Theorem 8. If $T$ has odd diameter at least seven and each vertex of its base is adjacent to a positive even number of leaves, then $T$ is 0-rotatable. Additionally, If $T$ has even diameter at least eight and each vertex of its base is adjacent to an even number of at least 4 leaves, then $T$ is 0-rotatable.

Outline of the proof. The technique used in this proof is similar to the technique used in the proof of Theorem 7 .

\section{Acknowledgments}

This work was funded by FAPESP, grants 2014/16987-1, 2014/16861-8, 2015/03372-1, and NSERC grant 41705-2014 057082.

\section{References}

Broersma, A. J. and Hoede, C. (1999). Another equivalent of the graceful tree conjecture. Ars Combinatoria, 51:183-192.

Bussel, F. V. (2004). 0-Centred and 0-ubiquitously graceful trees. Discrete Mathematics, 277:193-218.

Chung, F. R. K. and Hwang, F. K. (1981). Rotatable graceful graphs. Ars Combinatoria, 11:239-250.

Gallian, J. A. (2015). A dynamic survey of graph labeling. The Electronic Journal of Combinatorics, DS6, 1-389.

Hrnčiar, P. and Haviar, A. (2001). All trees of diameter five are graceful. Discrete Mathematics, 233:133-150.

Rosa, A. (1967). On certain valuations of the vertices of a graph. Theory of Graphs (Internat. Sympos., Rome, 1966) Gordon and Breach, New York; Dunod, Paris, 349355.

Rosa, A. (1977). Labeling snakes. Ars Combinatoria, 3:67-73. 